\title{
Assessing cardiac pumping capability by exercise testing and inotropic stimulation
}

\author{
L B TAN, R J I BAIN, W A LITTLER \\ From the Department of Cardiovascular Medicine, University of Birmingham, East Birmingham Hospital, \\ Birmingham
}

SUMMARY In heart failure both functional capacity and prognosis are primarily determined by the degree of pump dysfunction. Although data on haemodynamic function at rest may indicate impaired cardiac function, they do not assess the capacity of the heart to respond to stress. Maximal bicycle ergometry and incremental intravenous inotropic stimulation in 31 patients with moderately severe heart failure were evaluated as methods of stressing the heart to determine cardiac pumping capability, which is defined as the cardiac power obtained during maximal stimulation. There was good agreement between the cardiac pumping capabilities assessed by these two methods. Maximal cardiac power output was better than maximal cardiac output and left ventricular stroke work index in representing cardiac pumping capability, because it was less dependent on the type of stimulation used during evaluation.

Inotropic challenge is at least as effective as exercise testing in assessing cardiac pumping capability in heart failure, and may be a better method in patients who find physical exercise difficult.

The primary defect in heart failure is the inability of the cardiac pump to deliver sufficient hydraulic energy to maintain the circulation required by the metabolism during exercise and, when failure becomes more severe, at rest as well. ${ }^{1}$ Variables such as left ventricular ejection fraction, cardiac index, and left ventricular stroke work index are often measured to assess the extent of the primary defect. ${ }^{23}$ Unfortunately, these variables correlated poorly with exercise capacity ${ }^{45}$ and prognosis in individual patients with heart failure. ${ }^{16}$

An alternative method of evaluating cardiac impairment has been proposed. ${ }^{7}$ It was suggested that cardiac power output is, from the point of view of fluid dynamics, the most logical variable to represent cardiac performance. Whether it reflects cardiac function more closely than the more commonly used variables, such as cardiac index and left ventricular stroke work index, remains to be seen. In theory, how good the heart is as a pump can be best represented by the maximum hydraulic power output achieved by the heart during maximal stimulation, and this value is termed the cardiac pumping capability. ${ }^{7}$ This

Requests for reprints to Dr L B Tan, Cardiac Department, John Radcliffe Hospital, Oxford OX3 9DU.

Accepted for publication 28 February 1989 variable, when determined by maximal inotropic stimulation, has been shown to be an accurate predictor of the prognosis of individual patients with severe pump failure. ${ }^{1}$ Because it is not known whether this pharmacologically determined cardiac pumping capability bears any relation to the functional capacity of the impaired heart during exercise, we compared cardiac pumping capability measured during incremental dobutamine infusion with that measured during symptom limited exercise.

\section{Patients and methods}

\section{PATIENTS}

We studied 31 patients ( 24 men and seven women) in moderate to severe heart failure in sinus rhythm (mean age of 59 years (range 44-69 years)). All except two patients (class IV) had symptoms in New York Heart Association class III. Dyspnoea on exertion was the predominant symptom in all patients, although two patients felt that fatigue was equally limiting. Patients with exercise induced angina or important ventricular arrhythmias were excluded. The mean (SD) left ventricular ejection fraction was $27 \cdot 2(11 \cdot 5) \%$. Two patients had dilated cardiomyopathy and the remainder had heart failure secondary to coronary artery disease. All the patients had had heart failure for more than four months before 
evaluation and none had a myocardial infarction in the six months before study. All patients gave informed consent to the study, which was approved by the ethical subcommittee of the East Birmingham Hospital.

\section{EXPERIMENTAL PROTOCOL}

An electrocardiograph, thermodilution Swan-Ganz catheters, and arterial cannulas were used to measure heart rate, right atrial pressure, pulmonary arterial and pulmonary wedge pressures, systemic arterial pressure, and cardiac output, which was measured in triplicate. Baseline resting haemodynamic data were measured every 20 minutes until the consecutive data did not vary by more than $10 \%$. Haemodynamic data were also collected during exercise. Patients exercised in a semierect position (at $45-60^{\circ}$ to the horizontal) on a bicycle ergometer, starting at 10$25 \mathrm{~W}$ and increasing in steps of 10-25 W every three minutes. The workloads were selected so that the patients reached peak exercise in less than 10 minutes. The exercises were terminated by exhaustion, dyspnoea, or leg muscle fatigue, or by important ventricular arrhythmia. None of these patients had to stop exercising because of arrhythmia. The exercise tests were performed in the postabsorptive state at about the same time of day, and four hours after the ingestion of diuretics. After patients had rested for four hours, we gave increasing doses of dobutamine starting at $2.5 \mu \mathrm{g} / \mathrm{kg} / \mathrm{min}$ and increasing by 2.5 or 5.0 $\mu \mathrm{g} / \mathrm{kg} / \mathrm{min}$ every 5-10 minutes to a maximum of $15-$ $30 \mu \mathrm{g} / \mathrm{kg} / \mathrm{min}$. The maximum dose was reached when there was no further increase in cardiac output and cardiac power output, or when a symptom became intolerable or important arrhythmia developed. One patient asked for the dobutamine infusion to be stopped (at a rate of $15 \mu \mathrm{g} / \mathrm{kg} / \mathrm{min}$ ) when considerable vasodilatation caused a sensation of intolerable hotness. In two patients the infusion ( $>10 \mu \mathrm{g} / \mathrm{kg} / \mathrm{min}$ ) was stopped because of ventricular bigeminy that prevented further increases in cardiac power output.

\section{Calculations}

From the haemodynamic data obtained, where all pressures were expressed as $\mathrm{mm} \mathrm{Hg}$, cardiac index (CI), left ventricular stroke work index (LVSWI), pulmonary vascular resistance (PVR), systemic vascular resistance (SVR), and cardiac power output (CPO) were calculated as follows: $\mathrm{CI}\left(\mathrm{l} \cdot \mathrm{min}^{-1} \cdot \mathrm{m}^{-2}\right)=$ cardiac output/body surface area; LVSWI (g.m.m ${ }^{-2}$ ) $=$ (mean systemic arterial pressure-mean pulmonary wedge pressure) $\times \mathrm{CI} \times 0 \cdot 136 /$ heart rate; PVR (dyn.s.cm ${ }^{-5}$ ) = (mean pulmonary arterial pressure-mean pulmonary wedge pressure) $\times$ 80/cardiac output; SVR (dyn.s. $\mathrm{cm}^{-5}$ ) = (mean systemic arterial pressure-mean right atrial pressure $) \times 80 /$ cardiac output; $C P O(W)=$ (mean systemic arterial pressure-mean right atrial pressure) $\times$ cardiac output $\times 2.2167 \times 10^{-3}$.

The maximum cardiac power output during exercise testing $\left(\mathrm{CPO}_{\mathrm{E}}\right)$ was that obtained when the patient was performing at maximal exercise workload. The maximum cardiac power output during dobutamine infusion $\left(\mathrm{CPO}_{\mathrm{D}}\right)$ was determined by the highest cardiac power output value obtained during the incremental dobutamine stimulation.

Statistical analysis was by Student's paired $t$ test. Differences were taken to be significant at $\mathbf{p}<0.05$. Results are expressed as mean (1 SD). We used the statistical method of Bland and Altman ${ }^{8}$ to assess the agreement between the two methods of evaluating cardiac pumping capability (dobutamine and exercise challenge).

Table Haemodynamic effects of peak exercise and dobutamine infusion (mean (SD))

\begin{tabular}{|c|c|c|c|c|c|c|c|c|}
\hline Variable & Control & & Dobutas & & Control & & Exercise & \\
\hline Heart rate/min & $75 \cdot 9$ & $(12 \cdot 3)$ & $109 \cdot 4$ & $(17 \cdot 6)$ & $78 \cdot 6$ & $(12 \cdot 6)$ & $104 \cdot 6$ & $(16.9)$ \\
\hline $\begin{array}{l}\text { Mean right atrial pressure } \\
\text { (mm Hg) }\end{array}$ & $7 \cdot 4$ & $(5 \cdot 8)$ & $5 \cdot 1$ & $(4 \cdot 4)$ & $7 \cdot 6$ & $(5 \cdot 7)$ & $10 \cdot 9$ & $(7 \cdot 7)^{\star \star}$ \\
\hline $\begin{array}{l}\text { Mean pulmonary arterial pressure } \\
(\mathbf{m m} \mathbf{H g})\end{array}$ & $27 \cdot 7$ & $(12 \cdot 4)$ & 26.9 & $(12 \cdot 4)$ & $27 \cdot 1$ & $(12 \cdot 3)$ & $37 \cdot 8$ & $(15 \cdot 7)^{\star \star}$ \\
\hline $\begin{array}{l}\text { Mean pulmonary artery wedge } \\
\text { pressure }(\mathrm{mm} \mathrm{Hg})\end{array}$ & $16 \cdot 0$ & $(9 \cdot 2)$ & $11 \cdot 4$ & $(7 \cdot 3)$ & $15 \cdot 5$ & $(9 \cdot 0)$ & 19.9 & $(10 \cdot 3)^{\star \star}$ \\
\hline $\begin{array}{l}\text { Mean systemic arterial pressure } \\
\quad(\mathrm{mm} \mathrm{Hg}) \\
\text { Cardiac index }\left(1 \cdot \mathrm{min}^{-1} \cdot \mathrm{m}^{-2}\right) \\
\text { Cardiac power output (W) }\end{array}$ & $\begin{array}{r}89 \cdot 5 \\
2.45 \\
0.83\end{array}$ & $\begin{array}{l}(13 \cdot 8) \\
(0.71) \\
(0.31)\end{array}$ & $\begin{array}{r}88 \cdot 7 \\
4 \cdot 32 \\
1 \cdot 41\end{array}$ & $\begin{array}{l}(13 \cdot 1) \\
(1.03) \\
(0.42)\end{array}$ & $\begin{array}{l}91 \cdot 2 \\
2 \cdot 44 \\
0 \cdot 82\end{array}$ & $\begin{array}{l}(12 \cdot 3) \\
(0 \cdot 73) \\
(0 \cdot 31)\end{array}$ & $\begin{array}{r}102 \cdot 7 \\
3 \cdot 76 \\
1 \cdot 38\end{array}$ & $\begin{array}{l}(15 \cdot 6)^{\star \star} \\
(1 \cdot 10)^{\star} \\
(0 \cdot 49)\end{array}$ \\
\hline $\begin{array}{l}\text { Pulmonary vascular resistance } \\
(\text { dyn.s.cm }\end{array}$ & 236 & $(160)$ & 174 & (126) & 238 & (179) & 231 & $(171)$ \\
\hline $\begin{array}{l}\text { Systemic vascular resistance } \\
\left(\text { dyn.s. } \mathrm{cm}^{-5}\right)\end{array}$ & 1600 & $(426)$ & 918 & (286) & 1626 & $(428)$ & 1163 & (318) \\
\hline $\begin{array}{l}\text { Left ventricular stroke work index } \\
\left(\mathrm{g} \cdot \mathrm{m} \cdot \mathrm{m}^{-2}\right)\end{array}$ & $34 \cdot 1$ & $(13 \cdot 8)$ & $43 \cdot 2$ & $(14 \cdot 4)$ & $34 \cdot 2$ & $(14 \cdot 7)$ & $42 \cdot 6$ & $(18 \cdot 5)$ \\
\hline
\end{tabular}

${ }^{\star} \mathrm{p}<0.05$ and ${ }^{\star \star} \mathrm{p}<0.01$ for dobutamine $v$ exercise. 


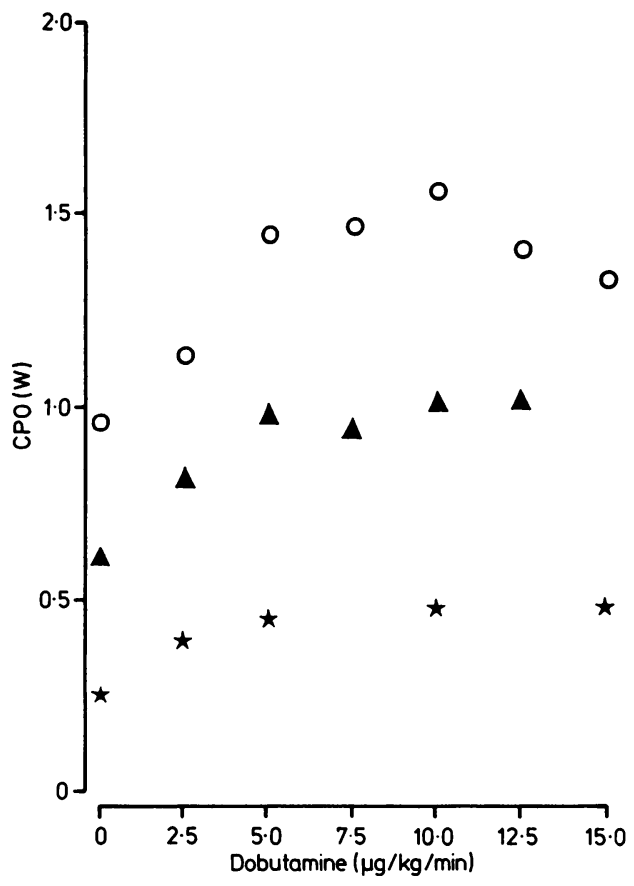

Fig 1 Cardiac power output (CPO) during incremental dobutamine infusion in three representative patients.

\section{Results}

The table compares the haemodynamic effects of dobutamine challenge and exercise. Both dobutamine and exercise increased heart rate, cardiac index, cardiac power output, and left ventricular stroke work index, and the peak values of these variables (except cardiac index) produced by both types of stimulation were not significantly different. Right and left ventricular filling pressures were reduced by dobutamine, but increased by exercise. Although dobutamine tended to reduce pulmonary and systemic vascular resistance more than exercise, resulting in lower pulmonary and systemic arterial pressures and higher cardiac output, similar left ventricular stroke work index and cardiac power output were obtained during both stresses.

Figure 1 shows cardiac power output during incremental dobutamine infusion in three representative patients. The increases in cardiac power output were more prominent at the lower doses of dobutamine, from 0 to $7.5 \mu \mathrm{g} / \mathrm{kg} / \mathrm{min}$. Above $10 \mu \mathrm{g} / \mathrm{kg} / \mathrm{min}$ the increases in cardiac power output with increments of dobutamine tended to reach a plateau. These patients had not been exposed to dobutamine and had not developed tolerance to it. When tolerance developed (for example after more than 24 hours of dobutamine infusion at $>10 \mu \mathrm{g} / \mathrm{kg} / \mathrm{min}$ ),
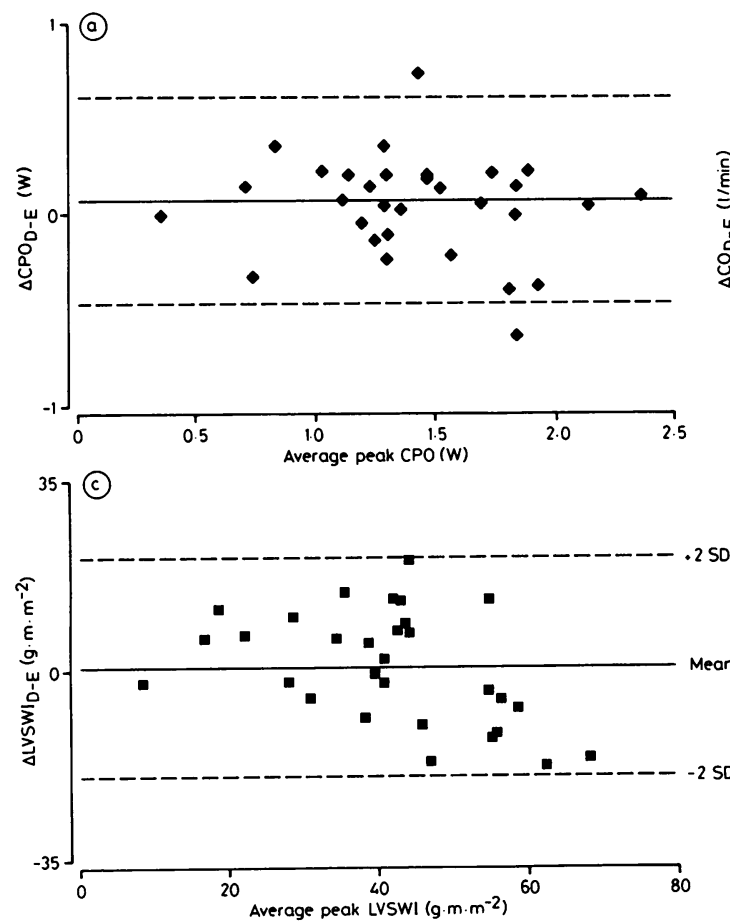

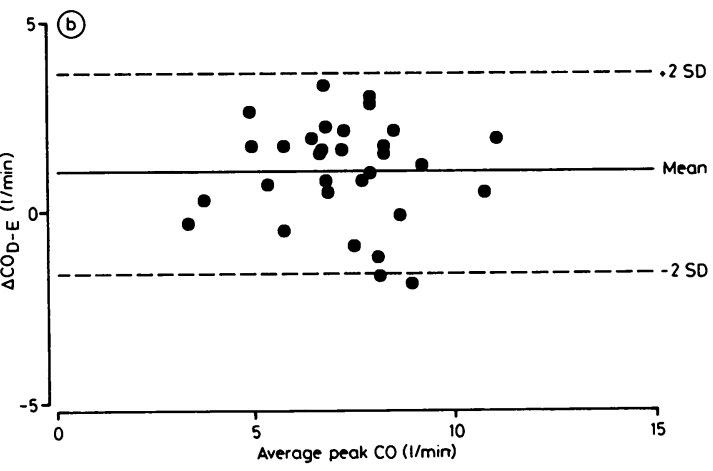

Fig 2 Differences of peak values of variables representing cardiac pump performance obtained during dobutamine (D) challenge and during exercise $(E)$ plotted against the average of the corresponding peak values. (a) Difference between maximal cardiac power output during exercise $\left(C P O_{E}\right)$ and that during dobutamine infusion $\left(C P O_{D}\right)$ plotted against the average values of paired $C P O_{D}$ and $C P O_{E} ;(b)$ change in cardiac output $\left(\mathrm{CO}_{D}-\mathrm{CO}_{E}\right)$ plotted against the average values of $C O_{D}$ and $C O_{E} ;(c)$ change in left ventricular stroke work index ( $\left.L V S W I_{D}-L V S W I_{E}\right)$ plotted against the average values of $L V S W I_{D}$ and $L V S W I_{E}$. 
higher doses of dobutamine (for example $>20 \mu \mathrm{g} / \mathrm{kg}$ / $\mathrm{min}$ ) were required to attain the peak cardiac power output. $\mathrm{CPO}_{\mathrm{D}}$ was unambiguously identified for each patient because values either reached a plateau at high doses or showed a peak followed by a decline.

The statistical method of Bland and Altman ${ }^{8}$ showed good agreement between the cardiac pumping capability as evaluated by measuring peak cardiac performance (represented by peak cardiac power output) during maximal dobutamine challenge and maximal exercise (fig 2a). When cardiac output was used to represent cardiac performance (fig $2 b$ ) the scatter of the points around the mean was similar, with the coefficient of variation of the differences (SD of differences/mean of the average peak values) being 0.19 in both assessments. However, the mean difference between $\mathrm{CPO}_{\mathrm{D}}$ and $\mathrm{CPO}_{\mathrm{E}}$ was closer to zero than that between $\mathrm{CO}_{\mathrm{D}}$ and $\mathrm{CO}_{\mathrm{E}}$. There were more points above than below zero in fig $2 \mathrm{~b}$. In other words, the peak cardiac output measured during dobutamine challenge tended to be greater than that measured during exercise. In fig $2 c$ the differences between left ventricular stroke work index measured by the two methods were plotted against the corresponding average left ventricular stroke work index. Like fig 2a, the mean value of the differences was close to zero. However, the scatter of the points about the mean in fig $2 c$ (coefficient of variation $=0.24$ ) was greater than those in figs $2 a$ and $b$ (coefficient of variation $=0 \cdot 19$ ).

\section{Discussion}

EVALUATION AT REST $\boldsymbol{v}$ DURING EXERCISE The heart of a patient in cardiac failure has limited functional capacity. Measurement of cardiac performance at basal resting states is helpful in detecting the presence of cardiac impairment (for example the low left ventricular ejection fraction of $27(12) \%$ and cardiac index of $2.5(0.7) 1 / \mathrm{min} / \mathrm{m}^{2}$ in these patients), but it often fails to differentiate the degree of functional cardiac failure. ${ }^{45} \mathrm{~A}$ patient's functional ability should not be assessed from haemodynamic measurements made only at rest.

To determine how well a heart functions as a pump during stress its response to maximal stimulation must be assessed. Clinically, the most important form of stimulation is physical exercise. At rest the cardiac power output of a normal subject is approximately $1 \mathrm{~W}$, and at maximal exercise it is approximately $6 \mathrm{~W} .^{7}$ The average (SD) cardiac power output of patients in this study was $0.8(0.3) W$ at rest and $1.4(0.5) W$ during maximal exercise, indicating a considerable reduction in cardiac pumping capability. Thus haemodynamic measurement during stress gives a clearer indication of failing cardiac function than haemodynamic evaluation at rest.

\section{CARDIAC PERFORMANCE EVALUATED BY}

CARDIAC POWER OUTPUT, CARDIAC OUTPUT, AND LEFT VENTRICULAR STROKE WORK INDEX The data shown in figs $2 a$ and $b$ suggest that cardiac power output is a better variable to represent cardiac pumping capability than cardiac output. For similar degree of cardiac stimulation, cardiac pumping capability during dobutamine challenge tended to be overestimated if cardiac output were used as the indicator of cardiac performance. This was because at peak stimulation blood pressure was significantly lower with dobutamine challenge than with exercise (table). There was a tendency for dobutamine to produce greater systemic arterial vasodilatation than exercise. Any drug that causes appreciable vasodilatation is liable to exaggerate cardiac output because the heart's capacity for generating pressure is converted into a capacity for generating flow.

Like cardiac power output, left ventricular stroke work index accounts for the capacity of the heart to generate both pressure and flow. So we were not surprised to find that the scatters of the differences above and below zero in fig $2 \mathrm{c}$ were almost equal. Left ventricular stroke work index is energy output per stroke whereas cardiac power output is energy output per unit time. Thus unlike cardiac power output, left ventricular stroke work index does not contain the factor heart rate. When heart rate is held constant these two measures of cardiac pumping capacity are equal. As far as maintaining the circulation is concerned, it does not matter how much hydraulic energy per stroke the heart delivers into the circulation, as long as the total delivered per unit time is sufficient to replenish the energy lost in the vasculature, and able to maintain organ perfusion and metabolism in tissues. Thus for any two hearts with the same limitation in generating stroke work, the one that is capable of a greater increase in heart rate would be able to impart more hydraulic energy into the vasculature and thus maintain a higher level of circulation and metabolism. Hence from the theoretical point of view, cardiac power output would be expected to be a better functional representation of cardiac pumping capability than left ventricular stroke work index. In patients with ischaemic cardiac failure, it is not uncommon to see excessive or inadequate chronotropic responses to exercise. The greater scatter of the points in fig $2 c$ than in fig $2 a$ suggests that the chronotropic responses of the hearts to dobutamine and exercise were different. Also, in fig $2 c$, peak left ventricular stroke work index was apparently higher with dobutamine challenge than with exercise when values were $<35$ g.m.m $\mathrm{m}^{-2}$ but the 
converse was true for higher values $\left(>50\right.$ g.m.m $\mathrm{m}^{-2}$ ). This implies that the use of left ventricular stroke work index as a measure of cardiac pumping capability depends on the mode of stimulation used and whether such stimulation can induce an appropriate chronotropic response.

\section{PHARMACOLOGICAL AGENTS TO STIMULATE THE} HEART

Dobutamine was chosen as the stimulating agent primarily because it was the most commonly used intravenous positive inotropic agent in the treatment of heart failure in our coronary care unit. As an adrenergic agonist it resembles the activity of endogenous catecholamines. ${ }^{910}$ One concern is that, as there is appreciable $\beta$ adrenoceptor down regulation in severe heart failure, ${ }^{11}$ adrenergic agonists may not be the ideal agent to use. The combined use of dobutamine with a phosphodiesterase inhibitor ${ }^{12}$ may circumvent this problem, but the combined arrhythmogenic effects of these drugs have not been ascertained. Furthermore, it may be argued that when patients exercise they have to rely on the cardiac response to adrenergic drive to augment their cardiac performance. Hence, from the functional point of view, the amount of cardiac reserve available via adrenergic stimulation is that which is of clinical and functional relevance.

\section{A COMPARISON OF PHARMACOLOGICAL AND} PHYSIOLOGICAL STIMULATION

This study showed that cardiac pumping capability measured during dobutamine challenge correlated well with that measured during symptom limited bicycle exercise testing, but there are advantages and disadvantages with both methods. The disadvantage of exercise testing lies in the difficulty in determining the true maximum exercise. It has been proposed that one way of identifying this point is to use the plateau of oxygen consumption as maximum exercise is approached, ${ }^{1314}$ but this proposal has been challenged. ${ }^{15}$ In contrast, $\mathrm{CPO}_{\mathrm{D}}$ can be easily identified during incremental dobutamine infusion (fig 1). Furthermore, since $\mathrm{CPO}_{\mathrm{D}}$ can be unambiguously determined (fig 1), it would be reasonable to propose that a necessary (but not sufficient) condition which must be satisfied before it can be claimed that the point of maximum exercise has been reached, is the attainment of $\mathrm{CPO}_{\mathrm{E}}$ that is greater than $\mathrm{CPO}_{\mathrm{D}}$.

Different exercise protocols may stress the heart differently, for example, more rapid increases in workload lead to earlier attainment of the maximum workload and a greater value of $\mathrm{CPO}_{\mathrm{E}}{ }^{716}$ During incremental dobutamine infusion, however, the ventricular filling pressures tend to decrease, which implies that $\mathrm{CPO}_{\mathrm{D}}$ might have been higher had it been possible to increase the preload concomitantly. Both exercise and incremental dobutamine infusion are potentially arrhythmogenic, and the occurrence of frequent ventricular extrasystoles or tachycardia tends to limit the maximal cardiac power output obtained.

Dobutamine challenge may be the best method of assessing cardiac pumping capacity particularly when the patient is unable or unwilling to undergo maximal exercise testing. Invasive techniques are not always needed because non-invasive techniques of measuring cardiac output are now available (for example Doppler echocardiography). ${ }^{17}$

\section{METHODS OF DETERMING PROGNOSIS}

Direct and indirect indicators of cardiac function have been used as prognostic indices in heart failure. Most of the direct indicators of cardiac function have been shown to give some measure of the prognosis in different patient groups, ${ }^{1819}$ but the considerable overlap of values between survivors and nonsurvivors limited the usefulness of these variables as discriminators in individual patients. ${ }^{16}$ Unfortunately, cardiac function was evaluated at basal resting states in these studies. An indirect measure of cardiac function that uses peak oxygen uptake during maximal exercise was shown to be a good prognostic index. ${ }^{20}$ The reason for this is that peak oxygen uptake is obtained during maximal stress. In other words, it is an indirect measure of the cardiac pumping capacity. However, it is also dependent on other factors as well, notably the regional distribution of blood flow and the way that exercising skeletal muscle consumes oxygen, and these factors are not known to influence prognosis unless the peripheral tissues become ischaemic at rest in terminal heart failure. Hence, a more direct measure of cardiac pumping capacity would be expected to be a better indicator of prognosis. ${ }^{1}$

The serum concentration of noradrenaline is also an indirect marker of cardiac dysfunction, and it has also been shown to be quite a good indicator of prognosis. ${ }^{21} 22$ The higher the noradrenaline concentration in severe heart failure the more likely is downregulation of the $\beta$ adrenoceptors. ${ }^{11}$ The evaluation of cardiac responsiveness to adrenergic stimulation (for example, dobutamine infusion) combines the information on cardiac pumping capacity and the sympathetic neurohormonal state of a patient with heart failure. ${ }^{1}$ It is therefore not surprising that this method of assessing prognosis is much more accurate than other methods of determining prognosis. What was not clear from the previous study was whether cardiac pumping capacity determined by dobutamine challenge is related to functional cardiac pumping capacity. This study showed a good agreement 
between the pharmacologically determined cardiac pumping capacity and physiologically determined cardiac pumping capacity. We can infer from this that the cardiac pumping capacity assessed by dobutamine challenge is a true indication of the functional capacity of the impaired heart during exercise. We therefore recommend that whenever a patient requires inotropic support, an incremental infusion of dobutamine should be given because the measurement of cardiac pumping capacity obtained will give important information on prognosis, which will help in deciding the future management of the patient.

The concept of evaluating cardiac function by cardiac pumping capacity is new. Its clinical utility is based on the understanding that measurement of cardiac function in the basal unstressed states is uncertain because of the variable degrees of activation of neurohormonal compensatory mechanisms on the one hand and on the other the variable states of somnolence or excitation of the patient during measurement. To avoid ambiguity, evaluation must be performed at maximal stress. The results of this study suggest that pharmacological stress is as good as physiological stress. The variable chosen to represent cardiac performance at this maximal point must not only reflect some isolated aspects of cardiac function (for example myocardial contractility) but also the overall integrity of the cardiac pumping. We found that cardiac power output was a better indicator of overall cardiac performances than cardiac index and left ventricular stroke work index.

We thank Mrs Elsie Gale and her team of technicians who helped to perform this study.

\section{References}

1 Tan LB. Cardiac pumping capability and prognosis in heart failure. Lancet 1986;ii:1360-3.

2 Braunwald E. Assessment of cardiac function. In: Braunwald E, ed. Heart disease. 3rd ed. Philadelphia: WB Saunders, 1988:449-70.

3 Arnold SB, Byrd RC, Meister W, et al. Long-term digitalis therapy improves left ventricular function in heart failure. $N$ Engl J Med 1980;303:1443-8.

4 Benge W, Litchfield RL, Marcus ML. Exercise capacity in patients with severe left ventricular dysfunction. Circulation 1980;60:955-9.

5 Franciosa JA, Park M, Levine TB. Lack of correlation between exercise capacity and indexes of resting left ventricular performance in heart failure. $\mathrm{Am} \mathrm{J}$ Cardiol 1981;47:33-9.

6 Smith WM. Epidemiology of congestive heart failure. Am J Cardiol 1985;55:3A-8A.

7 Tan LB. Clinical and research implications of new concepts in the assessment of cardiac pumping per- formance in heart failure. Cardiovasc Res 1987;21: 615-22.

8 Bland JM, Altman DG. Statistical methods for assessing agreement between two methods of clinical measurement. Lancet 1986;i:307-10.

9 Tuttle RR, Mills J. Dobutamine: development of a new catecholamine to selectively increase cardiac contractility. Circ Res 1975;36:185-96.

10 Robie NW, Nutter DO, Moody C, McNay JL. In vivo analysis of adrenergic receptor activity of dobutamine. Circ Res 1974;34:663-71.

11 Fowler MB, Laser JA, Hopkins GL, Minhobe W, Bristow MR. Assessment of the beta-adrenergic receptor pathway in the intact failing human heart: progressive receptor down-regulation and subsensitivity to agonist response. Circulation 1986; 74:1290-302.

12 Gage J, Rutman H, Lucido D, LeJemtel TH. Additive effects of dobutamine and amrinone on myocardial contractility and ventricular performance in patients with severe heart failure. Circulation 1986;74:367-73.

13 Weber KT, Kinasewitz GT, Janicki JS, Fishman AP. Oxygen utilization and ventilation during exercise in patients with chronic cardiac failure. Circulation 1982;65:1213-23.

14 Weber KT, Janicki JS. Cardiopulmonary exercise testing: physiologic principles and clinical application. Philadelphia: WB Saunders, 1986.

15 Wilson JR, Fink LI, Dunkman WB, Jones RA. Use of maximal bicycle exercise testing with respiratory gas analysis to assess exercise performance of patients with congestive heart failure secondary to coronary artery disease or to idiopathic dilated cardiomyopathy. Am J Cardiol 1986;58:601-6.

16 Lipkin DP, Canepa-Anson R, Stephens MR, PooleWilson PA. Factors determining symptoms in heart failure: comparison of fast and slow exercise tests. $\mathrm{Br}$ Heart J 1986;55:439-45.

17 Christie J, Sheldahl LM, Tristani FE, Sagar KB, Ptacin MJ, Wann S. Determination of stroke volume and cardiac output during exercise: comparison of twodimensional and Doppler echocardiography, Fick oximetry and thermodilution. Circulation 1987;76: $539-47$.

18 Likoff MJ, Chandler SL, Kay HR. Clinical determinants of mortality in chronic congestive heart failure secondary to idiopathic dilated or ischaemic cardiomyopathy. Am J Cardiol 1987;59:634-8.

19 Franciosa JA, Wilen M, Ziesche S, Cohn JN. Survival in man with severe chronic left ventricular failure due to either coronary heart disease or idiopathic dilated cardiomyopathy. Am J Cardiol 1983;51:831-6.

20 Willens HJ, Blevins RD, Wrisley D, Antonishen D, Reinstein D, Rubenfire $M$. The prognostic value of functional capacity in patients with mild to moderate heart failure. Am Heart $J$ 1987;114:377-82.

21 Rector TS, Olivari MT, Levine TB, Francis GS, Cohn JN. Predicting survival for an individual with congestive heart failure using plasma norepinephrine concentration. Am Heart J 1987;114:148-52.

22 Cohn JN, Levine TB, Olivari MT, et al. Plasma norepinephrine as a guide to prognosis in patients with chronic congestive heart failure. $N$ Engl J Med 1984;311:819-23. 